\title{
Adaptive change-point mixed models applied to data on outpatient tetracycline use in Europe
}

\author{
Girma Minalu ${ }^{1}$, Marc Aerts ${ }^{1}$, Samuel Coenen ${ }^{2,3}$, Ann Versporten ${ }^{3}$, \\ Arno Muller ${ }^{3}$, Niels Adriaenssens ${ }^{2,3}$, Philippe Beutels ${ }^{4}$, Geert Molenberghs ${ }^{1,5}$, \\ Herman Goossens ${ }^{3}$ and Niel Hens ${ }^{1,4}$ \\ ${ }^{1}$ Interuniversity Institute for Biostatistics and statistical Bioinformatics (I-BIOSTAT), \\ Hasselt University, Belgium \\ ${ }^{2}$ Vaccine \& Infectious Disease Institute (VAXINFECTIO), Centre for General Practice, \\ University of Antwerp, Belgium \\ ${ }^{3}$ Laboratory of Medical Microbiology, Vaccine \& Infectious Diseases Institute (VAXINFECTIO), \\ University of Antwerp, Belgium \\ ${ }^{4}$ Centre for Health Economics Research and Modeling Infectious Diseases (CHERMID), \\ Centre for the Evaluation of Vaccination, Vaccine and Infectious Disease Institute \\ (VAXINFECTIO), University of Antwerp, Belgium \\ ${ }^{5}$ Interuniversity Institute for Biostatistics and statistical Bioinformatics (I-BIOSTAT), \\ Catholic University of Leuven, Belgium
}

\begin{abstract}
In this paper, we propose a change-point mixed model to assess the change in the trend of outpatient antibiotic use in a Bayesian framework, where the change-points are unknown parameters of the model. Model selection using DIC indicates that the data supports the model with a countryspecific change-point. The location of the change-points may be related to points in time where public health strategies aiming at increasing the awareness of the public to a more rational use of antibiotics or targeting to reduce overconsumption of antibiotics were initiated.
\end{abstract}

Key words: amplitude; antibiotic use; change-point model; non-linear model; phase shift; seasonal variation

Received January 2012; revised March 2013; accepted March 2013

\section{Introduction}

Antibiotics are drugs that inhibit or abolish the growth of bacteria. Antibiotic resistance is a major European and global public health problem and international efforts

Address for correspondence: Marc Aerts, Interuniversity Institute for Biostatistics and statistical Bioinformatics (I-BIOSTAT), Hasselt University, Campus Diepenbeek, Agoralaan 1, B-3590, Diepenbeek, Belgium. E-mail: marc.aerts@uhasselt.be 


\section{Girma Minalu et al.}

are needed to counteract the emergence of resistance. The increase in resistance rate of many important pathogens to currently most available antibiotics has now been recognized as a universal health hazard and potentially life-threatening problem. A large number of studies strongly suggest that this increase is directly related to the actual use of antibiotics. Antibiotic use is increasingly recognized as the main driver for resistance and differential selection pressure of antibiotic agents may be responsible for some of the observed differences (Goossens et al., 2005; Davey et al., 2008; Huttner et al., 2010).

Specific actions such as campaigns aimed at the public and general practitioners appeared essential, because antibiotic use in outpatients accounts for the main part of the overall antibiotic usage ( $>90 \%$ ) (Goossens et al., 2005; Adriaenssens et al., 2011). Campaigns directed to the public in order to $(i)$ inform about antibiotic resistance and to warn about the medical and general health issues related to the inappropriate use of antibiotics and (ii) foster the patient-physician and patient-pharmacist dialogue about the appropriate use of antibiotics, will increase the awareness of the public to a more rational use of antibiotics. In some European countries (e.g., in Belgium, France, Germany, Greece, Iceland, Italy, Luxembourg, Portugal, Spain and United Kingdom), campaigns were planned as part of a national strategy to reduce resistance to antimicrobial drugs. These strategies also included measures to promote appropriate use of antimicrobial drugs in hospitals, long-term care facilities and the agricultural sector (Huttner et al., 2010).

Longitudinal data on outpatient antibiotic use were available from 27 European countries for the period 1997-2009 within the European Surveillance of Antimicrobial Consumption (ESAC) project (Adriaenssens et al., 2011; Coenen et al., 2011; Minalu et al., 2011). Given that repeated measures were taken for each country, intra-country correlation has to be taken into account when analyzing the data. The main objective of the study is to develop an appropriate statistical model to assess the significance of country-specific trends in Europe and to identify possible change-points, while accounting for country-specific global use as well as seasonal effects.

In common regression, time series or longitudinal data analysis, the outcome variable is modelled as a linear function of explanatory variables and/or time. Sometimes it may happen that the relationship between the outcome and some explanatory variables and/or time is non-smooth (non-differentiable), showing one or more points where the effect on the response changes abruptly. These points are called breakpoints, change-points, transition-points or switch-points. To estimate the changepoints, Bayesian (Smith, 1975; Carlin et al., 1992; Lange et al., 1992; Kiuchi et al., 1995; Slate and Turnbull, 2000; Ghosh and Vaida, 2007; Dominicus et al., 2008) or likelihood (Pastor and Guallar, 1998; Hall et al., 2000; Hall et al., 2003; Muggeo, 2003; Jacqmin-Gadda et al., 2006; Hens et al., 2010) methods may be used.

Within the likelihood framework, Pastor and Guallar (1998) used a two-segmented logistic regression model to estimate a change-point in the context of dose-response analysis in epidemiological studies. Muggeo (2003) proposed an approach to estimate broken line models reducing the problem to a linear framework. Jacqmin-Gadda 
et al. (2006) proposed a random change-point model which combines a piecewise polynomial mixed model with a random change-point for the evolution of the cognitive test and a log-normal model depending on the random change-point for the time to dementia.

A fully Bayesian hierarchical structure for a mixed effects segmented regression model with one change-point was considered by Slate and Turnbull (2000), and applied to large data sets concerning prostate specific antigen as a serial marker for prostate cancer. Ghosh and Vaida (2007) proposed a change-point model with one random change-point for the analysis of longitudinal CD4 T-cell counts for HIV infected subjects following highly active antiretroviral treatment. And Dominicus et al. (2008) studied a Bayesian random change-point model with one random changepoint to capture variability in measures of cognitive function. Hall et al. (2003) compared the Bayesian approach with the likelihood approach for modelling cognitive function over time, and pointed out that the Bayesian method has an advantage over the likelihood method in that it does not require all subjects to have the same change-point.

In this paper, an adaptive Bayesian linear spline model is proposed, where the number of knots (change-points) and their location are data driven and determined by the deviance information criterion (DIC). The presence and the location of the change-points is data driven and can vary across countries as random change-points. Latent country-specific indicators allow the model to switch off the change-points for particular countries.

The application of the model may yield new and important insights in the evolution of outpatient antibiotic use in Europe. We employ a fully Bayesian approach. The models are implemented in R using the R-package R2WinBUGS (Sturtz et al., 2005). The programs used for the analyses are available upon request from the first author. The program used to fit the change-point model with one unknown common change-point, one country-specific random change-point and a country-specific latent indicator for the change-point is included in Appendix III.

The paper is organized as follows. In Section 2, we describe the data on the total outpatient antibiotic use analyzed in the paper. In Section 3, we describe the models, the prior distributions for the parameters and we discuss how model comparison was applied. Results are presented in Section 4. Finally, discussions and concluding remarks are included in Section 5.

\section{Outpatient antibiotic use data}

Quarterly ESAC-NET data on total outpatient antibiotic use from 27 European countries were collected for the period 1997-2009 within ESAC-NET, an international network of surveillance systems. The methods of data collection and processing for the ESAC-NET project have been described in detail elsewhere (Adriaenssens et al., 2011; Coenen et al., 2011), and are also available on the ESAC-NET website (www.esac.ua.ac.be). Antibiotic use data is expressed as the number of defined 


\section{Girma Minalu et al.}

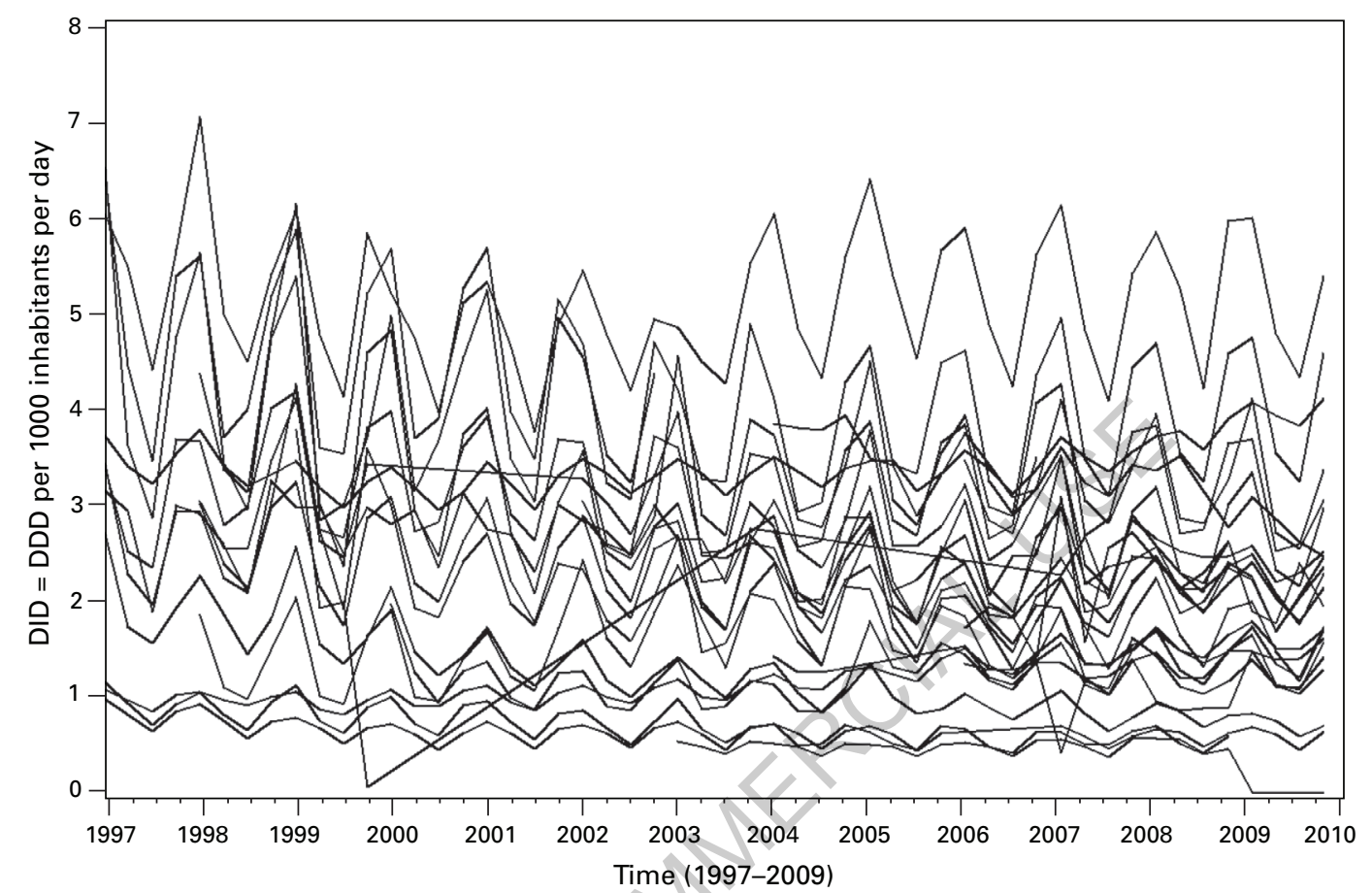

Figure 1 Observed country-specific evolutions for the quarterly use of tetracycline expressed in DID in 27 European countries

daily doses (DDD) per 1000 inhabitants per day (DID). This paper focuses on the outpatient use of tetracycline for the period 1997-2009, with the observed countryspecific trends for the quarterly tetracycline use in DID shown in Figure 1.

As can be seen in Figure 1, there is variability across repeated measurements from the same country (i.e., within-country variability) as well as variability between countries (i.e., between-country variability), which suggests that country-specific intercepts and slopes should be incorporated into the model to account for heterogeneity across countries. The longitudinal profiles show clear seasonal variation of outpatient tetracycline use in all countries, with upward peaks in the winter season. Thus, a non-linear model needs to be adopted to take the seasonality into account. From the longitudinal profiles it can clearly be seen that countries with higher tetracycline use at the baseline (in 1997) have a higher amplitude (higher seasonal variation). Figure 1 also shows that not all longitudinal profiles are complete for all countries. Some profiles start later in time and others show intermediate missing parts. As the missingness mechanism is assumed to be missing completely at random (MCAR), all analyses were based on all available cases. 


\section{Adaptive change-point models}

We start by introducing a non-linear mixed model with a sinusoidal component over time to account for the seasonal variation. We extend the non-linear mixed model by including fixed and random change-points to identify possible changes in the trend of tetracycline use in DID. We extend the existing approaches for random change-point models (e.g., Kiuchi et al., 1995; Ghosh and Vaida, 2007; Dominicus et al., 2008) by a general model building procedure where the number of knots and their location are data driven, and by taking into account a country-specific seasonal variation. The change-point models were also extended by including country-specific latent indicators, allowing the model to switch off the change-points for particular countries. All models are fitted in a fully Bayesian paradigm.

\subsection{Non-linear mixed model}

We applied the non-linear mixed model (3.1) to model the use of tetracycline in DID. An extension with known common change-points, unknown common change-points and country-specific random change-points is then considered. The non-linear mixed model is formulated as

$$
Y_{i j}=\left(\beta_{0}+b_{0 i}\right)+\left(\beta_{1}+b_{1 i}\right) t_{i j}+\left(\beta_{0}^{S}+b_{0 i}^{S}+\beta_{1}^{S} t_{i j}\right) \sin \left(\omega t_{i j}+\delta\right)+\varepsilon_{i j},
$$

where $Y_{i j}$ is the total outpatient tetracycline use in DID for country $i(i=1,2, \ldots, N)$ at time points $t_{i j}\left(j=1,2, \ldots, n_{i}\right), n_{i}$ is the number of observations from the $i$ th country, time $=1$ corresponds to the start of the study (first quarter of 1997), $\boldsymbol{\beta}=\left(\beta_{0}, \beta_{1}, \beta_{0}^{S}, \beta_{1}^{S}, \delta\right)$ is a vector of fixed effects, $\beta_{0}$ is the intercept, $\beta_{1}$ is the regression coefficient describing the marginal linear time trend $(t), \beta_{0}^{S}$ is the fixed amplitude, $\beta_{1}^{S}$ is the amplitude varying over time, $\omega$ (in radians) is the frequency which is a known constant $(=2 \pi / T)$ where $T(=4)$ is the period for the sine curve, $\delta$ (in radians) is the phase shift or phase angle which is an unknown parameter, $\boldsymbol{b}_{i}=\left(b_{0 i}, b_{1 i}, b_{0 i}^{S}\right)$ is the country-specific vector of random effects where $b_{0 i}$ is the country-specific random intercept, $b_{1 i}$ is the country-specific random slope for time and $b_{0 i}^{S}$ is the countryspecific random slope for amplitude and we assume $b_{i} \sim N(0, D)$. The matrix $D$ is a general covariance matrix with elements $d_{i j}=d_{j i} . \boldsymbol{\varepsilon}_{i}$ is an $n_{i}$-dimensional vector of unexplained error terms $\varepsilon_{i j}$. It is usually assumed that all $\boldsymbol{\varepsilon}_{i}$ are independent and normally distributed with mean vector zero and covariance matrix $\boldsymbol{\Sigma}_{i}$. Often, $\boldsymbol{\Sigma}_{i}$ is assumed equal to $\sigma_{\varepsilon}^{2} \boldsymbol{I}_{n_{i}}$, where $\boldsymbol{I}_{n_{i}}$ is the $n_{i}$-dimensional identity matrix.

Since no convergence was obtained when using an unstructured covariance matrix for the random effects, a diagonal covariance matrix was used. 


\section{Girma Minalu et al.}

\subsection{Adaptive change-point model}

Since there is no prior knowledge on the number of change-points, we gradually build up the model by first considering a change-point model with a known common change-point and then extending it by including unknown common and countryspecific random change-points.

A general mixed model with country-specific mean can be written as

$$
\begin{aligned}
& Y_{i j}=\mu_{i}\left(t_{i j}\right)+\varepsilon_{i j}, \quad i=1,2, \ldots, N ; j=1,2, \ldots, n_{i}, \\
& \mu_{i}\left(t_{i j}\right)=\mu_{i}^{T}\left(t_{i j}\right)+\mu_{i}^{S}\left(t_{i j}\right),
\end{aligned}
$$

where $Y_{i j}$ is the tetracycline use in DID for country $i$ at time points $t_{i j}, \mu_{i}^{T}\left(t_{i j}\right)$ is the trend component, $\mu_{i}^{S}\left(t_{i j}\right)$ is the seasonal component and $\varepsilon_{i j}$ is the measurement error which is assumed to be normally distributed with mean zero and constant variance $\sigma_{\varepsilon}^{2}$. The country-specific mean components $\mu_{i}^{T}\left(t_{i j}\right)$ and $\mu_{i}^{S}\left(t_{i j}\right)$ are modelled as

$$
\begin{aligned}
& \mu_{i}^{T}\left(t_{i j}\right)=\left(\beta_{0}+b_{0 i}\right)+\left(\beta_{1}+b_{1 i}\right) t_{i j}+\mu_{i}^{C P}\left(t_{i j}\right), \\
& \mu_{i}^{S}\left(t_{i j}\right)=\left(\beta_{0}^{S}+b_{0 i}^{S}+\beta_{1}^{S} t_{i j}\right) \sin \left(\omega t_{i j}+\delta\right),
\end{aligned}
$$

where $\mu_{i}^{C P}\left(t_{i j}\right)$ is a change-point component given by

$$
\mu_{i}^{C P}\left(t_{i j}\right)=\sum_{k=1}^{K}\left(\beta_{(k+1)}+b_{(k+1) i}\right)\left(t_{i j}-K_{k i}\right)_{+},
$$

where $x_{+}=\max (x, 0), K$ is the number of unknown change-points, $K_{k i}=C_{k}$ or $K_{k i}=C_{k}+c_{k i}$ or $K_{k i}=c_{k i}$ where $C_{k}$ denotes a global change-point and $c_{k i}$ a countryspecific random change-point. If $\mu_{i}^{C P}\left(t_{i j}\right)=0$ then there are no change-points and the model reduces to model (3.1).

Substituting equations (3.3) and (3.4) in equation (3.2) yields the model

$$
\begin{aligned}
Y_{i j}= & \left(\beta_{0}+b_{0 i}\right)+\left(\beta_{1}+b_{1 i}\right) t_{i j}+\sum_{k=1}^{K}\left(\beta_{(k+1)}+b_{(k+1) i}\right)\left(t_{i j}-K_{k i}\right)_{+} \\
& +\left(\beta_{0}^{S}+b_{0 i}^{S}+\beta_{1}^{S} t_{i j}\right) \sin \left(\omega t_{i j}+\delta\right)+\varepsilon_{i j},
\end{aligned}
$$

where the fixed effects $\beta_{0}, \beta_{1}, \beta_{0}^{S}, \beta_{1}^{S}, \omega$ and $\delta$, and the random effects $b_{0 i}, b_{1 i}$ and $b_{0 i}^{S}$ are defined as before, $K$ is the number of change-points, for $k=1,2, \ldots, K, \beta_{(k+1)}$ is the global difference in the linear trend before and after the change-point, $b_{(k+1) i}$ is the country-specific difference in the linear trend before and after the change-point and $\varepsilon_{i j}$ is an unexplained error term. Random effects for the global level of use, the trend effects, the amplitude of the seasonal effect and the location of the change-point are used to account for heterogeneity across countries. The number of change-points $K$ and the location of the change-point(s) are data driven. 
In Equation (3.5) all countries are assumed to have a change in the trend of tetracycline use in DID, but this might not be true because some countries might not have a change in the trend of tetracycline use. To relax this assumption, we extend (3.5) by including country-specific latent indicators for the change-points,

$$
\begin{aligned}
Y_{i j}= & \left(\beta_{0}+b_{0 i}\right)+\left(\beta_{1}+b_{1 i}\right) t_{i j}+\sum_{k=1}^{K}\left\{\left(\beta_{(k+1)}+b_{(k+1) i}\right)\left(t_{i j}-K_{k i}\right)_{+}\right\} I_{k i} \\
& +\left(\beta_{0}^{S}+b_{0 i}^{S}+\beta_{1}^{S} t_{i j}\right) \sin \left(\omega t_{i j}+\delta\right)+\varepsilon_{i j},
\end{aligned}
$$

where $I_{k i}$ is an unknown country-specific indicator for the change in the trend of tetracycline use in DID for country $i$ for the $k$ th change-point, $k=1,2, \ldots, K$ where $K$ is the number of change-points. Here, $I_{k i}=1$ if there is a change at knot $K_{k i}$ in the use of tetracycline over time in country $i$, or $I_{k i}=0$ if there is no change in the use of tetracycline over time in country $i$.

As there are no prior information on the number of change-points in the study, the number of change-points $K$ in Equations (3.5) and (3.6) has to be chosen prior to the data fitting, $k=1, \ldots, K$. We first start from the simplest model where there is only a known common change-point, i.e., $K=1$. We gradually extend the model by including a known and an unknown common change-point. And later, we extended the model by including an additional unknown common change-point. Next to the common change-points, country-specific random change-points have also been included in the model.

\subsection{Prior specification}

The following uninformative prior distributions were used for the fixed effects:

$\beta_{0}, \beta_{1}, \beta_{(k+1)}, \beta_{0}^{S}, \beta_{1}^{S}, \delta \sim \operatorname{Normal}(0,1000)$, independently where $k=1, \ldots, K$,

$$
\begin{aligned}
& C_{1} \sim \text { Uniform }(1,52), \\
& C_{2} \sim \text { Uniform }\left(C_{1}, 52\right) .
\end{aligned}
$$

The normal priors on $\beta_{0}, \beta_{1}, \beta_{(k+1)}, \beta_{0}^{S}, \beta_{1}^{S}$ and $\delta$ have large variances, expressing our lack of knowledge about the regression coefficients. For the random effects, a normal prior distributions was used:

$$
\begin{aligned}
b_{0 i} & \sim \operatorname{Normal}\left(0, \sigma_{b_{0}}^{2}\right), \\
b_{1 i} & \sim \operatorname{Normal}\left(0, \sigma_{b_{1}}^{2}\right), \\
b_{(k+1) i} & \sim \operatorname{Normal}\left(0, \sigma_{b_{(k+1)}}^{2}\right), \\
b_{0 i}^{S} & \sim \operatorname{Normal}\left(0, \sigma_{b_{0}^{S}}^{2}\right), \\
c_{k i} & \sim \operatorname{Normal}\left(C_{k}, \sigma_{c_{k}}^{2}\right) I(1,52) .
\end{aligned}
$$




\section{Girma Minalu et al.}

A uniform prior distribution over the total range of time was also assumed for the country-specific random change-point:

$$
c_{k i} \sim \operatorname{Uniform}(1,52) .
$$

The country-specific indicator for the $k$ th change-point $\left(I_{k i}\right)$ is Bernoulli-distributed with probability $P_{k}$, where the probability $P_{k}$ is beta-distributed with shape parameters $\alpha_{p}(=1)$ and $\beta_{p}(=1)$ :

$$
\begin{aligned}
I_{k i} & \sim \operatorname{dbern}\left(P_{k}\right), \\
P_{k} & \sim \operatorname{dbeta}(1,1) .
\end{aligned}
$$

The hyperparameters in the prior distributions were chosen so that the priors are uninformative. An independent inverse gamma distribution with a shape parameter $\alpha(=0.001)$ and a scale parameter $\beta(=0.001)$ was used for the variance parameters.

$$
\sigma_{b_{0}}^{2}, \sigma_{b_{1}}^{2}, \sigma_{b_{(k+1)}}^{2}, \sigma_{b_{0}^{s}}^{2}, \sigma_{c_{k}}^{2}, \sigma_{\varepsilon}^{2} \sim \operatorname{IGamma}(0.001,0.001) \text {, independently, }
$$

where $x \sim \operatorname{IGamma}(\alpha, \beta)$ means that $1 / x$ has the Gamma distribution with mean $\alpha / \beta$ and variance $\alpha / \beta^{2}$ (Ntzoufras, 2009).

\subsection{Model selection}

We use the DIC for model comparison (Spiegelhalter et al., 2002). The DIC can be represented as:

$$
D I C=p_{D}+\bar{D}
$$

DIC is a Bayesian equivalent to Akaike's information criterion (AIC) and consists of two components, a term that measures goodness-of-fit $(\bar{D}$, defined as the posterior expectation of the deviance) and a penalty term for model complexity ( $p_{D}$, defined as the difference between the posterior mean of deviance and the deviances evaluated at the posterior mean $\bar{\theta}$ of the parameters). $p_{D}=\bar{D}-D(\bar{\theta})$. The smaller the DIC, the better the fit (Spiegelhalter et al., 2002; Gelman et al., 2004; Ghosh and Vaida, 2007; Dominicus et al., 2008).

There has been and there still is discussion on Bayesian model selection in general and on the specification of the prior for model selection and hypothesis testing, related to the Jeffreys-Lindley paradox (Lindley, 1957). See, for instance, Spiegelhalter et al. (2002) in which pros and cons of several approaches to Bayesian model selection are discussed by the authors and several discussants. Posterior model probabilities and Bayes factors might be considered to represent the gold standard in fully Bayesian model determination, but these quantities are sensitive to the choice of prior distribution in the case of specifying a default prior under weak prior information (Overstall and Forster, 2010). Criterion-based methods such as BIC or DIC do not give posterior model probabilities, and as such the issue of default prior specification is avoided. Here we opted for DIC as a criterion for model selection. An in-depth analysis of this issue with a comparison of the performance and characteristics of 
different model selection criteria is considered beyond the scope of this paper. For more details and some recent developments on this issue related to Lindley's paradox, we refer to Casella et al. (2009), Mulder et al. (2009), Overstall and Forster (2010), and references therein.

Plummer (2008) provided a justification for the DIC by demonstrating the link between DIC and cross-validation. In his paper, DIC is shown to be an approximation to a penalized loss function based on the deviance, with a penalty derived from a cross-validation argument. This approximation is valid only when the effective number of parameters in the model is much smaller than the number of independent observations (i.e., $\left.p_{D}<<n\right)$. A corrected DIC, $D I C_{c}=\bar{D}+\sum_{i=1}^{n} p_{D_{i}} /\left(1-p_{D_{i}}\right.$ ), was suggested for generalized linear mixed models when the DIC cannot be justified as approximation to the penalized plug-in deviance. To the best of our knowledge, the use of the corrected DIC has not been studied for non-linear mixed models and requires further research. Therefore, we do not pursue its use in this paper.

The quarterly tetracycline use data was analyzed in Minalu et al. (2011) using the non-linear mixed model. The results of the non-linear mixed models were used as a starting value for the MCMC algorithm. And for the additional change-point parameters, the locations of campaigns or policy changes in antibiotic use in most European countries were used as starting values. To ensure adequate convergence all results were obtained using two chains of 110000 iterations, of which we discarded the first 10000 (burn-in) and the chain was then thinned to every 5th sample as there was autocorrelation for some parameters. Trace plots and the potential scale reduction $\hat{R}$ were used to check convergency of the MCMC algorithm (Gelman et al., 2004).

\section{Results}

We considered the following models, within the family (3.5):

Model 1: Non-linear mixed model without a change-point,

$$
\mu_{i}^{C P}\left(t_{i j}\right)=0
$$

Model 2: Non-linear mixed model with a known common change-point $\left(C_{1}=\right.$ 17),

$$
\mu_{i}^{C P}\left(t_{i j}\right)=\left(\beta_{2}+b_{2 i}\right)\left(t_{i j}-17\right)_{+},
$$

Model 3: Non-linear mixed model with a known common change-point $\left(C_{1}=\right.$ 29),

$$
\mu_{i}^{C P}\left(t_{i j}\right)=\left(\beta_{2}+b_{2 i}\right)\left(t_{i j}-29\right)_{+},
$$

Model 4: Non-linear mixed model with one unknown common change-point $\left(C_{1}\right)$, 


\section{Girma Minalu et al.}

$$
\mu_{i}^{C P}\left(t_{i j}\right)=\left(\beta_{2}+b_{2 i}\right)\left(t_{i j}-C_{1}\right)_{+},
$$

Model 5: Non-linear mixed model with two unknown common change-points $\left(C_{1}\right.$ and $\left.C_{2}\right)$,

$$
\mu_{i}^{C P}\left(t_{i j}\right)=\left(\beta_{2}+b_{2 i}\right)\left(t_{i j}-C_{1}\right)_{+}+\left(\beta_{3}+b_{3 i}\right)\left(t_{i j}-C_{2}\right)_{+},
$$

where ordering restriction was imposed for the common change-points (i.e., $C_{1}<$ $\mathrm{C}_{2}$ ).

Model 6: Non-linear mixed model with one country-specific random changepoint $\left(c_{i}\right)$,

$$
\mu_{i}^{C P}\left(t_{i j}\right)=\left(\beta_{2}+b_{2 i}\right)\left(t_{i j}-c_{i}\right)_{+},
$$

where the country-specific random change-point is centred around the unknown point $C_{1}$ and is restricted to lay within [1,52], $c_{i} \sim N\left(C_{1}, \sigma_{c}^{2}\right)(1,52)$.

Model 7: Non-linear mixed model with one country-specific random changepoint $\left(c_{i}\right)$,

$$
\mu_{i}^{C P}\left(t_{i j}\right)=\left(\beta_{2}+b_{2 i}\right)\left(t_{i j}-c_{i}\right)_{+},
$$

where the country-specific random change-points are $U(1,52)$ distributed.

Model 1 without a change-point is first extended with known common changepoints (Models 2 and 3). Because there were public campaigns in some of the European countries during the year 2000-01 (e.g., in Belgium, Germany and Greece) and during the year 2004-05 (e.g., in Portugal and United Kingdom), we used time $=17$ (first quarter of 2001) and time = 29 (first quarter of 2004) as known common change-points in the trend of tetracycline use in DID, respectively, in Model 2 and Model 3. Next, we estimate the change-points by including unknown common and/or country-specific random change-points (Models 4-7). The non-linear mixed model (Model 1) was extended by including a non-linear trend and secondly an amplitude varying non-linearly over time (expressed as $t_{i j}^{\alpha}$ ). As these extended models did not outperform the change-point models, we only presented the results of the original non-linear and the change-point models (Models 1-7). Various models with three change-points were applied too, but convergence could not be reached for any of these models.

For the unknown common change-points in Models 4-6, uniform prior distributions over the total range of time were used. A normal prior distribution with mean zero and variance $\sigma_{c_{k}}^{2}$ was used for the country-specific random change-point in Model 6, while in Model 7 a uniform prior distribution over the total range of time was assumed for the country-specific random change-point. A summary of the posterior distributions of the model parameters in Models 1-7 is given in Table 1 .

The results in Table 1 clearly indicate the need for one or more change-points. Indeed, Model 1 (no change-points) gets little support with the highest DIC $=391.6500$. Including a known common change-point reduces the DIC considerably (Models 2 


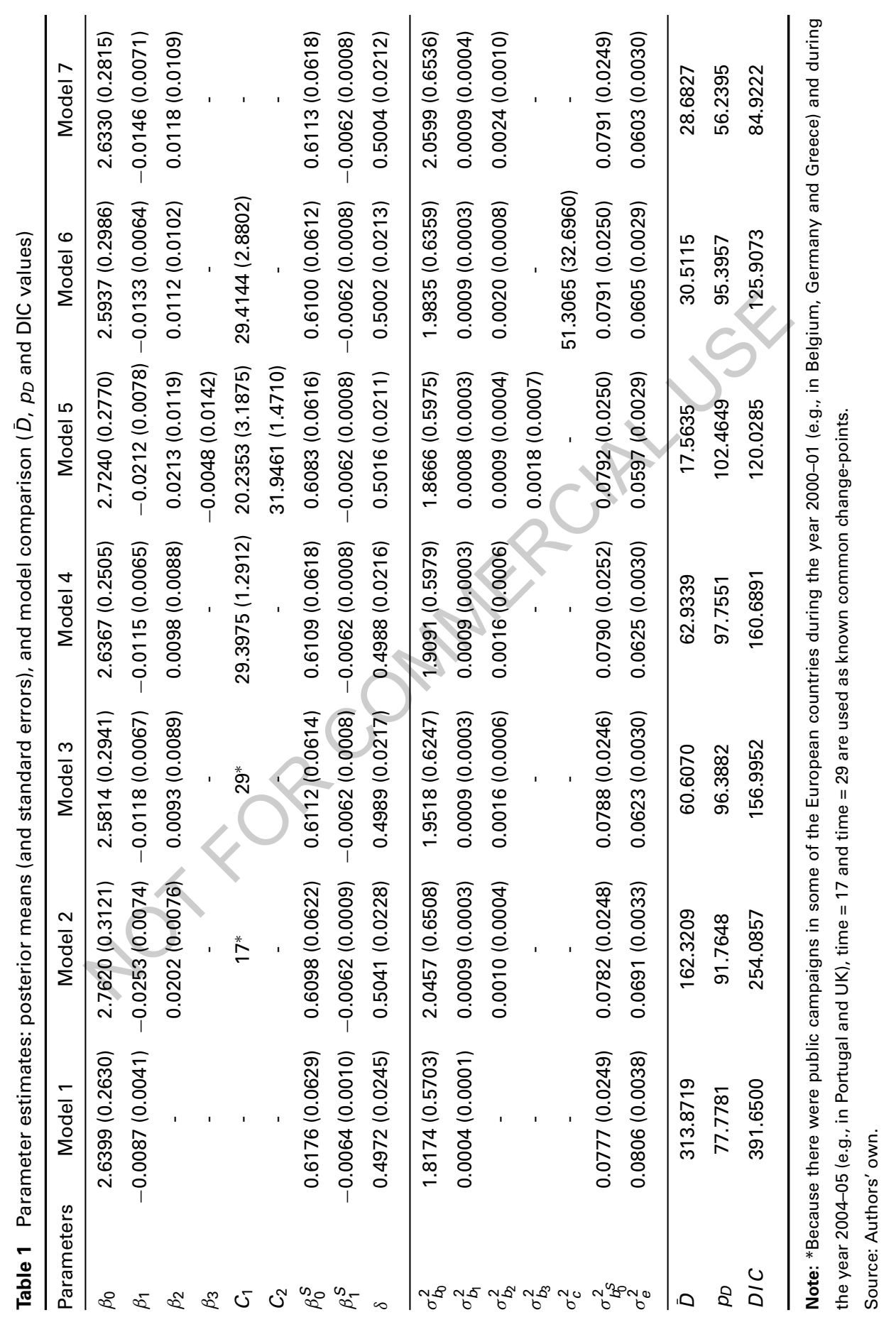




\section{Girma Minalu et al.}

and 3). There is no improvement when the known change-point 29 is replaced by an unknown common change-point (Model 4). There is, however, a further improvement when two unknown common change-points are included in the model (Model 5). In Models 2-5 all countries are assumed to have the same common change-point, while in Models 6-7 all countries have different change-points. Comparing Model 6 with Model 4 shows a reduction in DIC when including a countryspecific random change-point next to the global change-point. A large improvement is achieved when a uniform prior distribution over the total range of time was used for the country-specific random change-point (Model 7). Scatter plots of countryspecific estimates for the change-points in Models 6 and 7 are shown in Figures A1 and A2 of Appendix I.

The estimate for the unknown common change-point $\left(C_{1}\right)$ obtained from fitting Model 4 is 29.3975 (fourth quarter of 2003), which is quite close to the estimates for the common change-point obtained from fitting Model $6\left(C_{1}=29.4144\right)$. The average for the estimated country-specific random change-points in Model 7 is 28.7451, which is very close to the estimate for the unknown common change-points in Models 4 and 6. From Model 5, the estimate for the first common change-point $\left(C_{1}\right)$ is 20.2353 (fourth quarter of 2001) and 31.9461 (fourth quarter of 2004) for the second common change-point $\left(C_{2}\right)$.

The $95 \%$ quantile-based credible interval for $\beta_{1}(-0.0270,-0.0003)$ indicates that there is a significant decrease in the global trend of tetracycline use in DID. The credible intervals for $\beta_{0}^{S}$ and $\beta_{1}^{S}$ do not include zero, indicating a significant overall seasonal variation and a significant overall seasonal variation trend over time, respectively.

The estimated linear trend (dashed line), the estimated change-point model (solid line) from Model 7 and the observed average DID for Europe are shown in Figure 2. The predicted mean is based on the predicted outcomes from the posterior distribution of the country-specific random effects. Figure 2 indicates that the model describes the data very well.

Models 2-7 assume that there are one or more trend changes of tetracycline use in all countries, but for some countries it might be better to have only one or even no change-point. To allow a data-adaptive selection of the number and location of the country-specific change-points, we extend Models 4-7 by including a latent country-specific indicator $I_{k i}$ for the $k$ th change-point, $k=1,2, \ldots, K$ for country $i(i=1,2, \ldots, N)$.

Model 4*: Non-linear mixed model with one unknown common change-point $\left(C_{1}\right)$ and a country-specific indicator $I_{1 i}$,

$$
\mu_{i}^{C P}\left(t_{i j}\right)=\left\{\left(\beta_{2}+b_{2 i}\right)\left(t_{i j}-C_{1}\right)_{+}\right\} I_{1 i}
$$

where $I_{1 i}$ is an unknown country-specific indicator for the change in the trend of DID for country $i$. Here, $I_{1 i}=1$ if a change at $C_{1}$ in the use of tetracycline over time in country $i$ is needed, or $I_{1 i}=0$ if no change in the use of tetracycline over time in country $i$ is needed, 


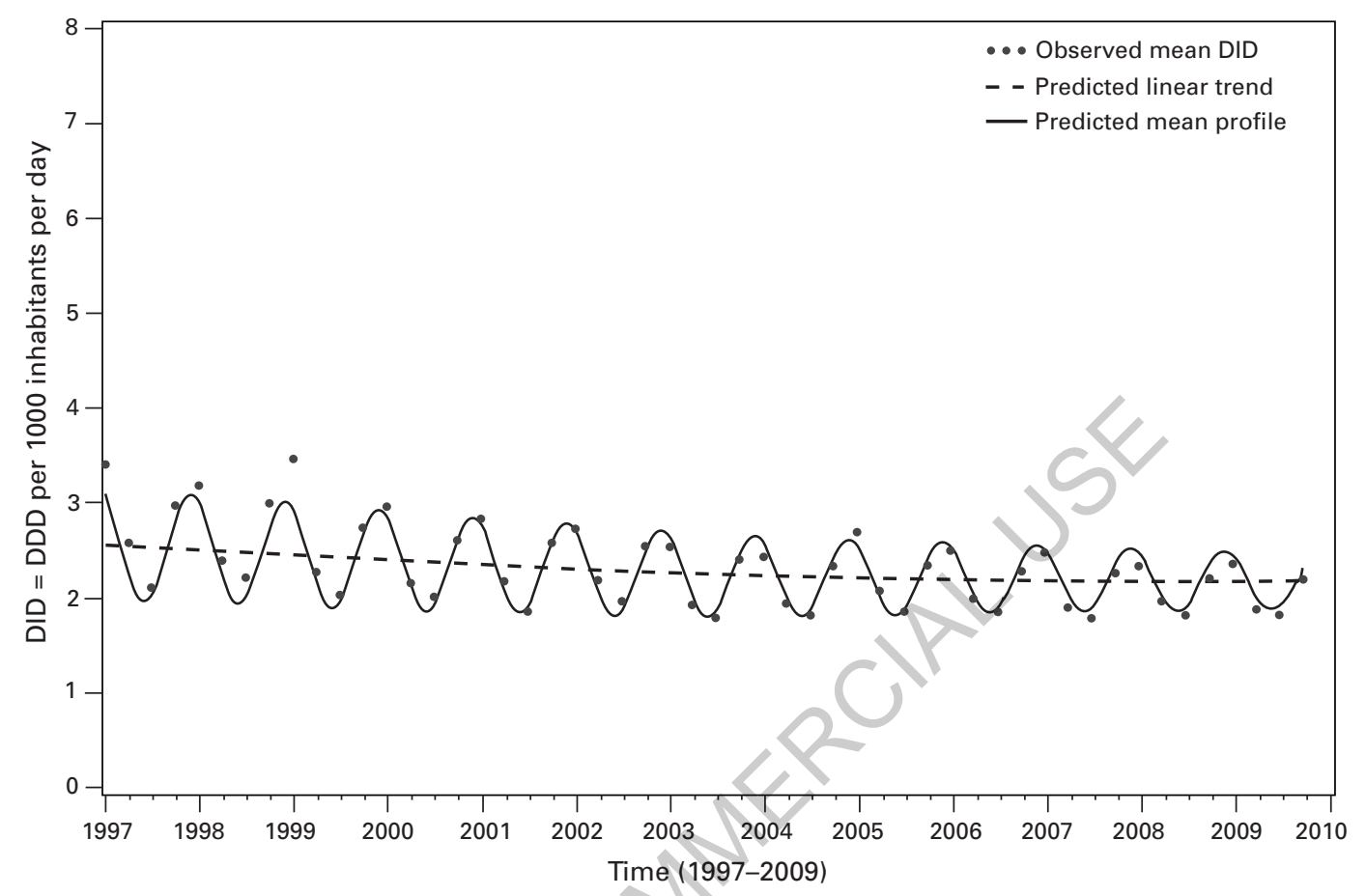

Figure 2 The observed mean DID (dots), the predicted mean profile (solid line) and the predicted linear trend (dashed line) obtained from fitting Model 7

Source: Authors' own.

Model 5*: Non-linear mixed model with two unknown common change-points $\left(C_{1}\right.$ and $\left.C_{2}\right)$ and two country-specific indicators $\left(I_{1 i}\right.$ and $\left.I_{2 i}\right)$,

$$
\mu_{i}^{C P}\left(t_{i j}\right)=\left\{\left(\beta_{2}+b_{2 i}\right)\left(t_{i j}-C_{1}\right)_{+}\right\} I_{1 i}+\left\{\left(\beta_{3}+b_{3 i}\right)\left(t_{i j}-C_{2}\right)_{+}\right\} I_{2 i},
$$

where ordering restriction was imposed for the common change-points (i.e., $C_{1}<$ $\mathrm{C}_{2}$ ).

Model $6^{*}$ : Non-linear mixed model with one country-specific random changepoint $\left(c_{i}\right)$ and a country-specific indicator $I_{1 i}$,

$$
\mu_{i}^{C P}\left(t_{i j}\right)=\left\{\left(\beta_{2}+b_{2 i}\right)\left(t_{i j}-c_{i}\right)_{+}\right\} I_{1 i},
$$

where the country-specific random change-point is centred around the unknown point $C_{1}$ and is restricted to lay within [1,52], $c_{i} \sim N\left(C_{1}, \sigma_{c}^{2}\right)(1,52)$.

Model 7*: Non-linear mixed model with a country-specific random changepoint $\left(c_{i}\right)$ and a country-specific indicator $I_{1 i}$, 


\section{Girma Minalu et al.}

Table 2 Parameter estimates: posterior means and standard errors, and model comparison: $\bar{D}, p_{D}$ and DIC values obtained from fitting Models $4^{*}$, 6* and $7^{*}$

\begin{tabular}{lccc}
\hline Parameters & Model $4^{*}$ & Model 6* & \multicolumn{1}{c}{ Model 7* } \\
\hline$\beta_{0}$ & $2.6488(0.2734)$ & $2.6618(0.2890)$ & $2.6322(0.2527)$ \\
$\beta_{1}$ & $-0.0120(0.0064)$ & $-0.0140(0.0066)$ & $-0.0139(0.0065)$ \\
$\beta_{2}$ & $0.0108(0.0105)$ & $0.0130(0.0111)$ & $0.0126(0.0126)$ \\
$C_{1}$ & $29.4560(1.2975)$ & $29.1115(2.8179)$ & - \\
$\beta_{0}^{S}$ & $0.6113(0.0630)$ & $0.6104(0.0613)$ & $0.6120(0.0615)$ \\
$\beta_{1}^{S}$ & $-0.0062(0.0008)$ & $-0.0062(0.0008)$ & $-0.0062(0.0008)$ \\
$\delta$ & $0.4985(0.0218)$ & $0.4999(0.0214)$ & $0.5002(0.0213)$ \\
$P_{1}$ & $0.8407(0.1222)$ & $0.8651(0.1091)$ & $0.8861(0.1008)$ \\
\hline$\sigma_{b 0}^{2}$ & $1.9527(0.6229)$ & $2.0181(0.6382)$ & $2.0580(0.6472)$ \\
$\sigma_{b 1}^{2}$ & $0.0009(0.0003)$ & $0.0009(0.0003)$ & $0.0009(0.0003)$ \\
$\sigma_{b 2}^{2}$ & $0.0018(0.0008)$ & $0.0022(0.0009)$ & $0.0026(0.0011)$ \\
$\sigma_{c}^{2}$ & - & $46.3954(30.8729)$ & - \\
$\sigma_{b 0}^{2} S$ & $0.0791(0.0252)$ & $0.0788(0.0248)$ & $0.0790(0.0251)$ \\
$\sigma_{e}^{2}$ & $0.0626(0.0030)$ & $0.0606(0.0030)$ & $0.0605(0.0030)$ \\
\hline $\bar{D}$ & 65.1738 & 32.7773 & 31.0169 \\
$p_{D}$ & 87.6091 & 92.3128 & 54.7426 \\
$D I C$ & 152.7831 & 125.0902 & 85.7595 \\
\hline
\end{tabular}

Note: ${ }^{*}$ Models 4, 6 and 7 are fitted with a country-specific latent indicator $I_{k i}$ Source: Authors' own.

$$
\mu_{i}^{C P}\left(t_{i j}\right)=\left\{\left(\beta_{2}+b_{2 i}\right)\left(t_{i j}-c_{i}\right)_{+}\right\} I_{1 i},
$$

where the country-specific random change points are $U(1,52)$ distributed.

The parameter estimates for all parameters in Models $4^{*}, 6^{*}$ and $7^{*}$ are given in Table 2. No convergence was obtained for Model $5^{*}$.

From the results given in Table 2 , Model $7^{*}$ has the lowest DIC value which is quite close to the DIC value of Model 7 (in Table 1). The parameter estimates given in Table 2 are also close to the corresponding parameter estimates given in Table 1. The parameter estimates for the country-specific latent indicators $I_{k i}$ are given in Table A1 in Appendix II. The posterior means for the change-point indicator $I_{k i}$ is greater than 0.5 for all countries, which indicates a change in the trend of tetracycline use for all countries.

The observed country-specific profiles and the predicted country-specific profiles from Model 7 for three selected countries (Iceland, Belgium and Austria) are shown in Figure 3. As can be seen from Figure 3, the predicted country-specific profiles follow closely the observed country-specific DID values. The bold dots indicate the estimated country-specific random change-points obtained from fitting Model 7.

A visual inspection of convergence diagnostics graphs for various model parameters showed that the posterior densities are smooth and unimodal shapes. The trace 


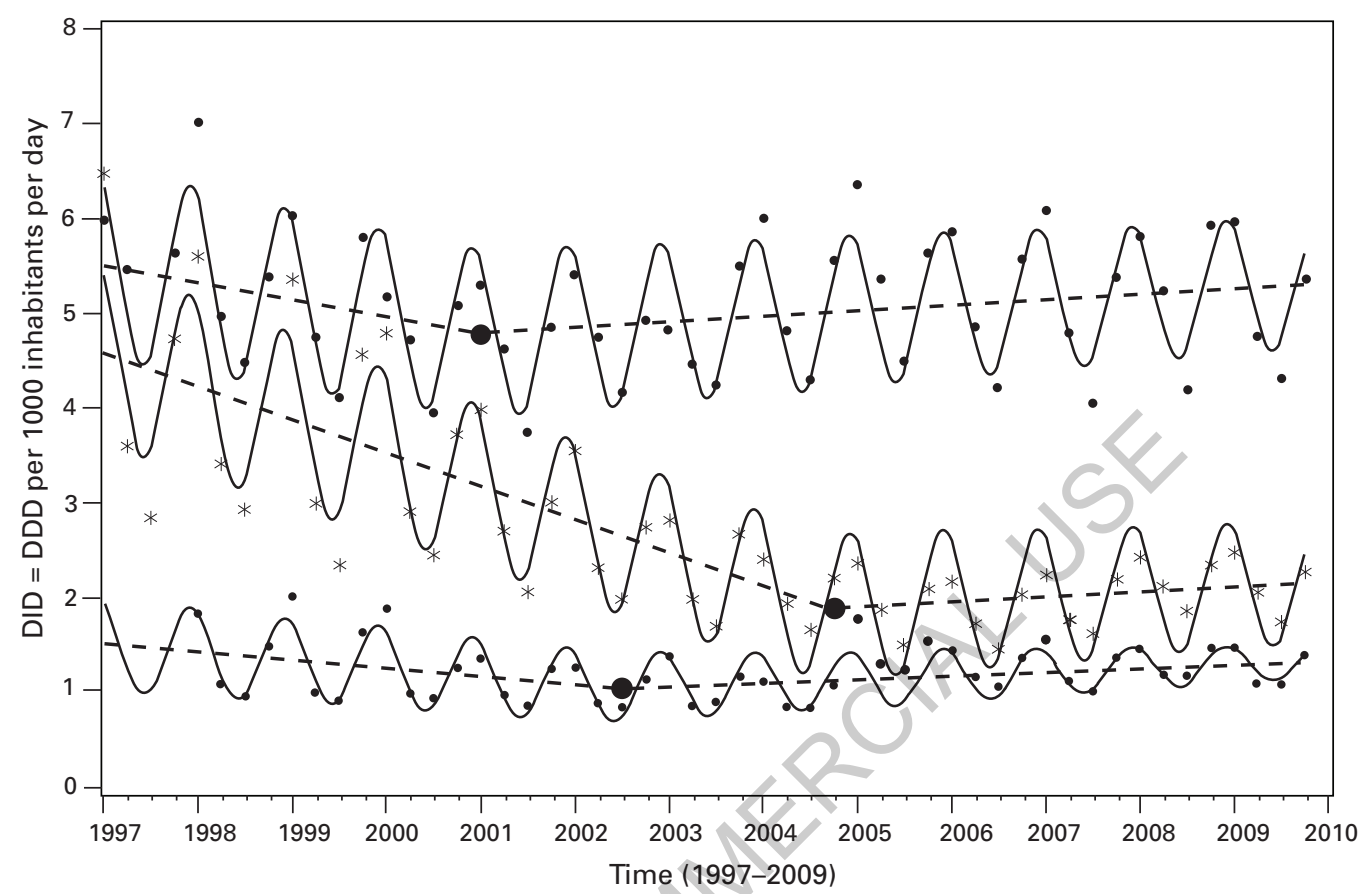

Figure 3 The observed country-specific DID (dots and stars), the predicted country-specific profiles (solid lines) and the country-specific predicted linear trends (dashed lines) obtained from fitting Model 7 for three selected countries (Iceland, Belgium and Austria from top to bottom)

Source: Authors' own.

plots indicate that chains appear to have reached a stationary distribution. The chain also has good mixing and is dense.

\section{Discussion}

This study was motivated by the need to assess the use of tetracycline in 27 European countries, to assess the change in the trend of tetracycline use over time, and to possibly relate any changes in antibiotics use due to campaigns and policy changes. The data have previously been analyzed based on a non-linear mixed model while taking into account the seasonal effects (Minalu et al., 2011). From the analysis, we have identified significant variation in total outpatient tetracycline use in Europe. Differences in tetracycline use between countries might be explained by variations in incidence of community acquired infections, culture and education, and differences in drug regulations and in the structure of the national pharmaceutical market (Goossens et al., 2005). 


\section{Girma Minalu et al.}

In this paper, we presented and discussed adaptive change-point Bayesian models to analyze the outpatient tetracycline use from 1997 to 2009 . We considered the non-linear mixed model extended with known common change-points, unknown common change-points and country-specific random change-points. The changepoint mixed model was also extended by including country-specific indicators for the change-points. A widely used statistic for comparing models in a Bayesian framework, the DIC, was used for model comparison. The model with country-specific changepoints (Model 7) has the lowest value of DIC. There is some controversy on which criterion to use to compare Bayesian models. Gelman et al. (2004) suggested $p_{V}=$ $\operatorname{Var}$ (Deviance)/2 as an estimate of the effective number of parameters in the model as an alternative to $p_{D}$. Note that using $p_{V}$ as an alternative measure of complexity, the change-point model with two unknown common change-points (Model 5) has the lowest DIC value.

The random change-point models have been applied in many applications (Kiuchi et al., 1995; Ghosh and Vaida, 2007; Dominicus et al., 2008). In this paper, we extended the existing approaches by a general model building procedure where the number of knots and their location are data driven. We also extended the previously proposed change-point models by taking into account a country-specific seasonal variation. The change-point models were also extended by including country-specific latent indicators, allowing the model to switch off the change-points for particular countries.

From the results obtained from fitting the change-point model with a countryspecific change-point (Model 7), there is a significant decrease in the trend of tetracycline use in DID. There is a significant seasonal variation in the use of tetracycline and also a significant seasonal variation trend over time.

The adaptive change-point models can be extended with more change-points. But for the tetracycline use data, convergence was not reached by including more than two common change-points or more than one country-specific random change-point. We have conducted a small-scale simulation study under different scenarios to investigate the change-point model in more detail. The results of this small-scale simulation study show that the change-point model with two (resp. three) change-points fits the data best when the data are generated under the change-point model with two (resp. three) change-points. As for the analyses of the case study, the change-point model with three (resp. four) change-points did not converge. This simulation experiment confirms that the convergence issues we encountered in our data application for the model with three or more change-points are very likely attributable to the absence of three or more change-points. 
Adaptive change-point mixed models applied to data on outpatient 269

\section{Appendix I: Estimates for the country-specific change-point}

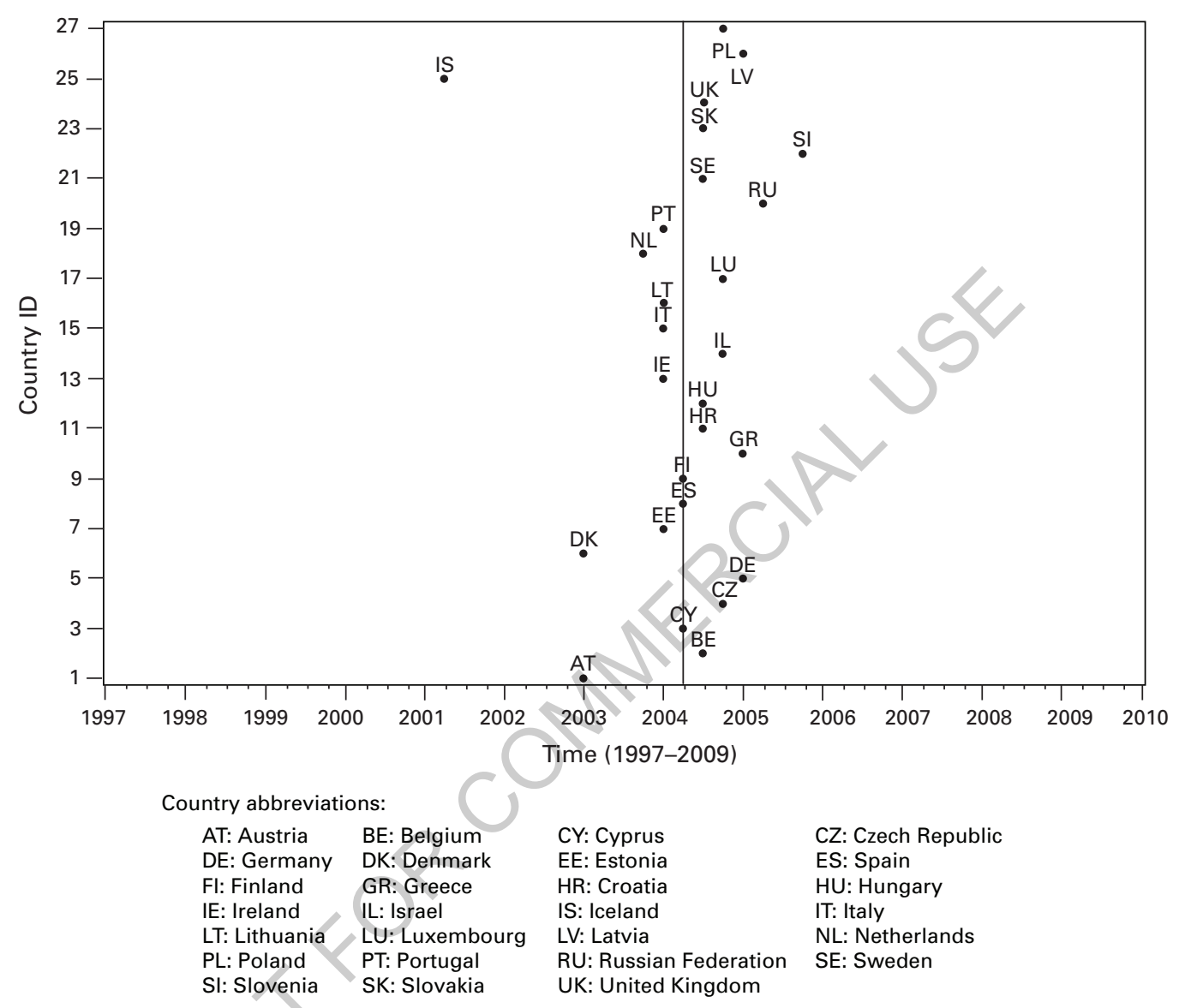

Figure A1 Scatter plot of estimates for the country-specific change-points obtained from fitting Model 6. The vertical line indicates the estimated global change-point.

Source: Authors' own. 
270 Girma Minalu et al.

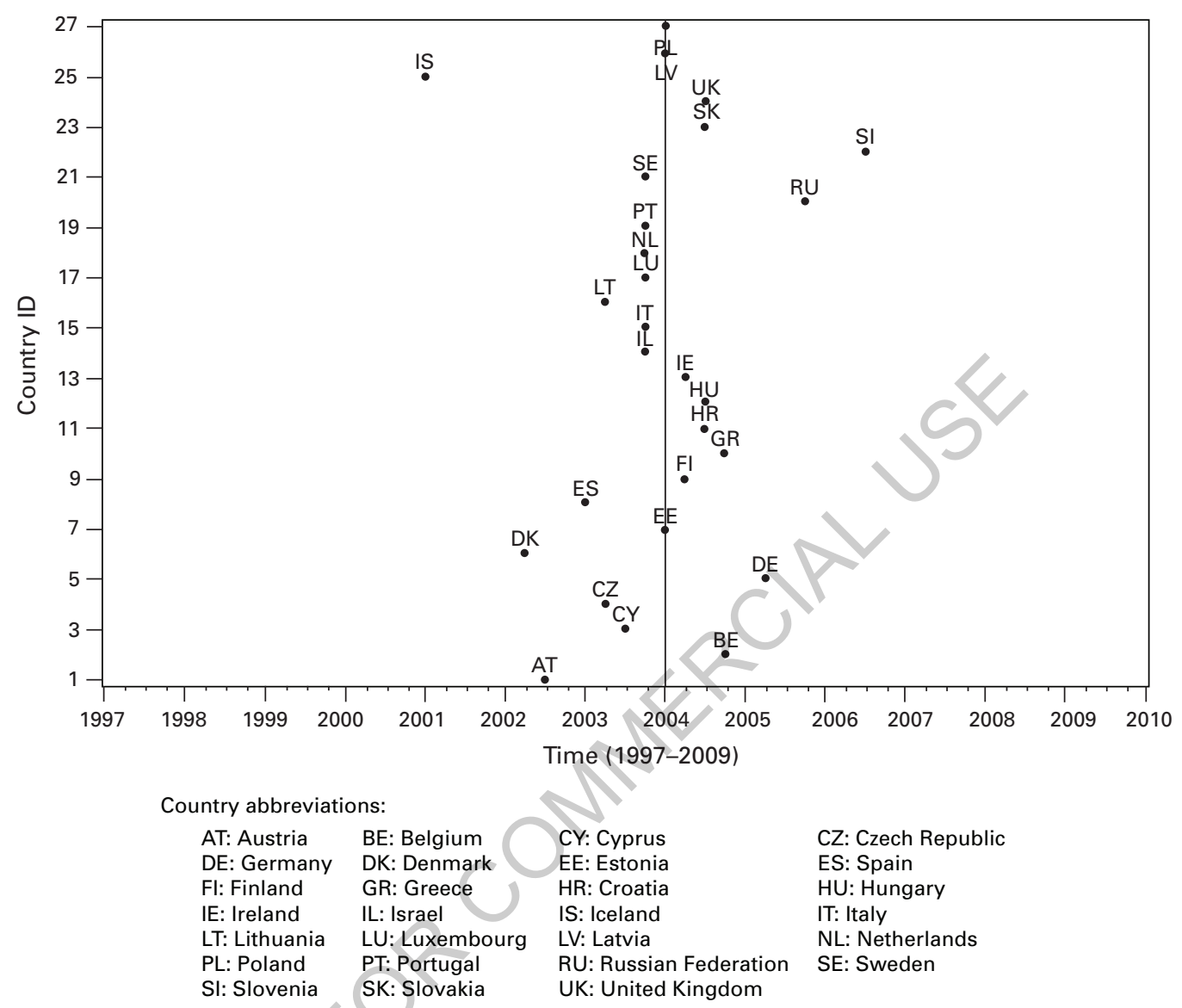

Figure A2 Scatter plot of estimates for the country-specific change-points obtained from fitting Model 7. The vertical line indicates the average for the estimated country-specific random change-points.

Source: Authors' own. 
Adaptive change-point mixed models applied to data on outpatient 271

\section{Appendix II: Estimates for the country-specific change-point indicators}

Table A1 Parameter estimates: posterior means (and standard errors) for the country-specific indicators $\left(I_{k i}\right)$ obtained from fitting Models $4^{*}, 6^{*}$ and $7^{*}$

\begin{tabular}{lcccc}
\hline Country & Parameters & Model $4^{*}$ & Model 6* & Model $7^{*}$ \\
\hline Austria & $I_{1}$ & $0.9516(0.2147)$ & $0.9693(0.1724)$ & $0.9629(0.1890)$ \\
Belgium & $I_{2}$ & $1.0000(0.0000)$ & $1.0000(0.0000)$ & $1.0000(0.0000)$ \\
Cyprus & $I_{3}$ & $0.8434(0.3635)$ & $0.8706(0.3356)$ & $0.8794(0.3257)$ \\
Czech Republic & $I_{4}$ & $0.8026(0.3980)$ & $0.8746(0.3311)$ & $0.8963(0.3048)$ \\
Germany & $I_{5}$ & $0.9696(0.1718)$ & $0.9785(0.1450)$ & $0.9740(0.1592)$ \\
Denmark & $I_{6}$ & $0.9054(0.2926)$ & $0.9194(0.2722)$ & $0.9241(0.2648)$ \\
Estonia & $I_{7}$ & $0.7104(0.4536)$ & $0.7515(0.4321)$ & $0.8006(0.3996)$ \\
Spain & $I_{8}$ & $0.6616(0.4732)$ & $0.7266(0.4457)$ & $0.8132(0.3898)$ \\
Finland & $I_{9}$ & $1.0000(0.0000)$ & $1.0000(0.0000)$ & $1.0000(0.0000)$ \\
Greece & $I_{10}$ & $0.8278(0.3776)$ & $0.8634(0.3434)$ & $0.8869(0.3167)$ \\
Croatia & $I_{11}$ & $0.9932(0.0819)$ & $0.9930(0.0834)$ & $0.9885(0.1066)$ \\
Hungary & $I_{12}$ & $0.9576(0.2016)$ & $0.9656(0.1823)$ & $0.9557(0.2057)$ \\
Ireland & $I_{13}$ & $0.8520(0.3551)$ & $0.8604(0.3466)$ & $0.8823(0.3222)$ \\
Israel & $I_{14}$ & $0.7719(0.4196)$ & $0.8041(0.3969)$ & $0.8389(0.3677)$ \\
Italy & $I_{15}$ & $0.8299(0.3757)$ & $0.8457(0.3613)$ & $0.8665(0.3401)$ \\
Lithuania & $I_{16}$ & $0.9705(0.1692)$ & $0.9773(0.1489)$ & $0.9756(0.1543)$ \\
Luxembourg & $I_{17}$ & $0.7520(0.4318)$ & $0.7994(0.4004)$ & $0.8286(0.3769)$ \\
Netherlands & $I_{18}$ & $0.9996(0.0194)$ & $0.9996(0.0212)$ & $0.9990(0.0308)$ \\
Portugal & $I_{19}$ & $0.8623(0.3446)$ & $0.8908(0.3119)$ & $0.8903(0.3125)$ \\
Russian Federation & $I_{20}$ & $0.8067(0.3949)$ & $0.8569(0.3502)$ & $0.9025(0.2967)$ \\
Sweden & $I_{21}$ & $0.5890(0.4920)$ & $0.6698(0.4703)$ & $0.8136(0.3894)$ \\
Slovenia & $I_{22}$ & $0.7065(0.4554)$ & $0.8022(0.3983)$ & $0.9013(0.2982)$ \\
Slovakia & $I_{23}$ & $1.0000(0.0071)$ & $1.0000(0.0000)$ & $0.9997(0.0166)$ \\
United Kingdom & $I_{24}$ & $0.9998(0.0158)$ & $0.9997(0.0180)$ & $0.9996(0.0200)$ \\
Iceland & $I_{25}$ & $0.9768(0.1505)$ & $0.9999(0.0087)$ & $1.0000(0.0000)$ \\
Latvia & $I_{26}$ & $0.7986(0.4011)$ & $0.8164(0.3872)$ & $0.8408(0.3659)$ \\
Poland & $I_{27}$ & $0.8389(0.3677)$ & $0.8594(0.3476)$ & $0.8678(0.3387)$ \\
\hline & & & & \\
\hline
\end{tabular}

Note: ${ }^{*}$ Models 4,6 and 7 are fitted with a country-specific latent indicator $I_{k i}$.

Source: Authors' own.

\section{Appendix III: $\mathbf{R}$ code}

The following WinBUGS code were used in R using the R-package R2WinBUGS to fit the change-point model with one unknown common change-point, one countryspecific random change-point and a country-specific latent indicator for the changepoint.

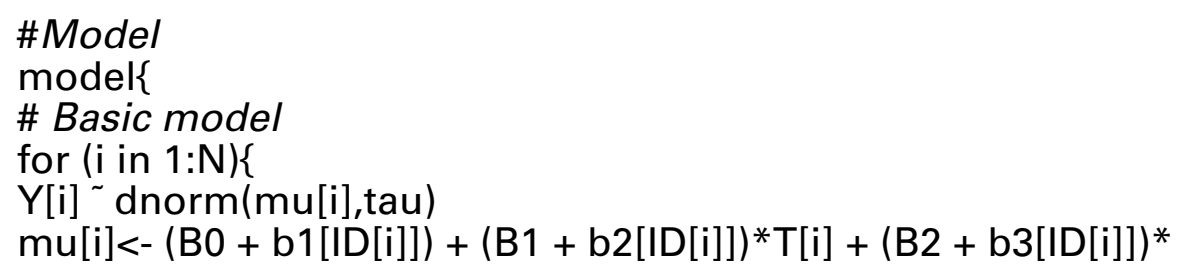


272 Girma Minalu et al.

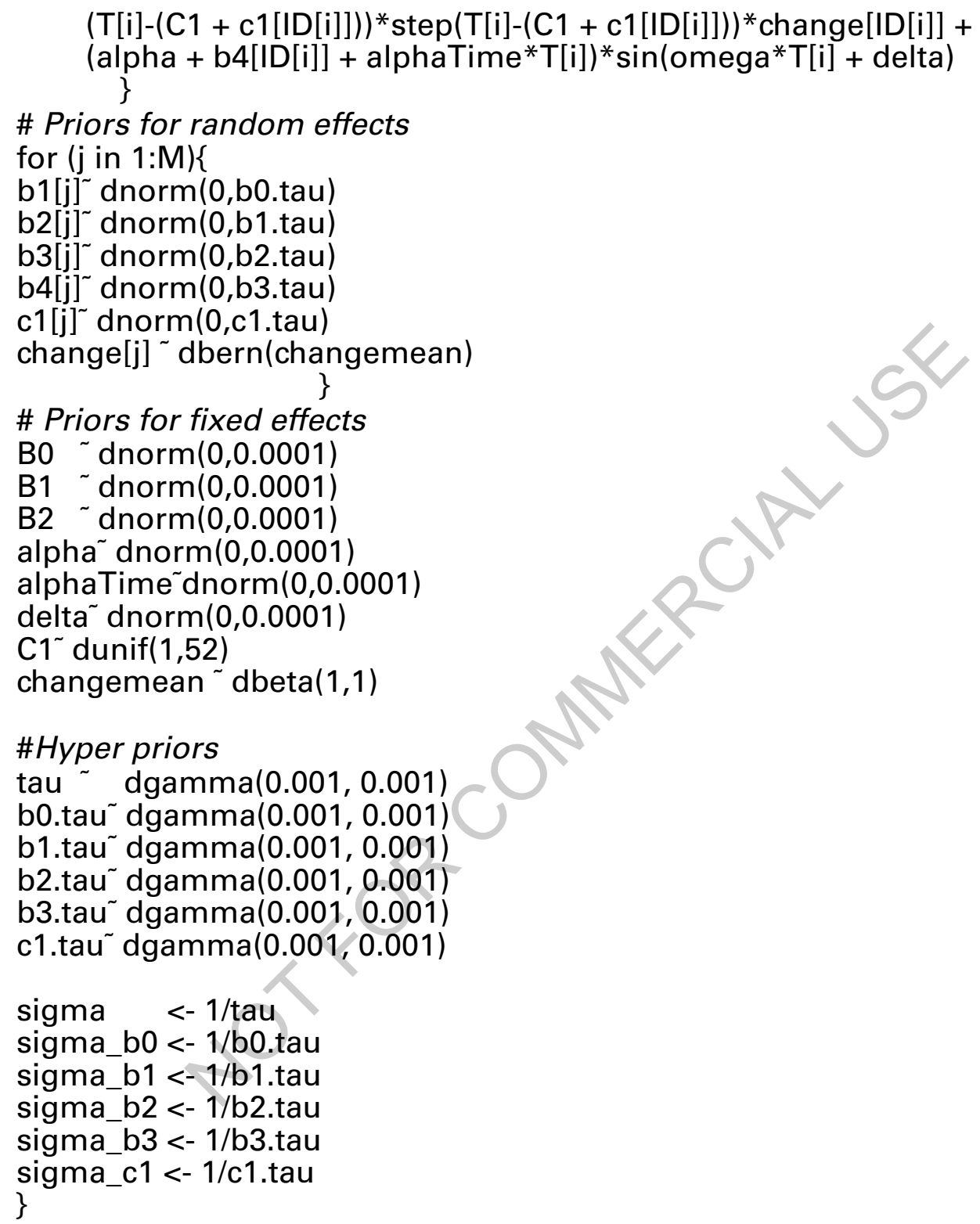

\section{Acknowledgements}

The authors would like to thank the Associate Editor and the reviewers for their valuable comments that have led to an improved version of the manuscript. We also gratefully acknowledge support from IAP research Network \#P6/03 of the Belgian 
Government (Belgian Science Policy). The 1997-2005 data collection was funded by a grant from DG SANCO of the European Commission (Grant Agreement 2003211), whereas the 2006-09 data collection was funded by the ECDC (Grant Agreement 2007/001).

\section{References}

Adriaenssens N, Coenen S, Versporten A, Muller A, Faes C, Vankerckhoven V, Aerts M, Hens N, Molenberghs G and Goossens $\mathrm{H}$ (on behalf of the ESAC Project Group) (2011) European Surveillance of Antimicrobial Consumption (ESAC): outpatient antibiotic use in Europe (1997-2009). Journal of Antimicrobial Chemotherapy, 66 Suppl. 6, vi3-vi12.

Carlin BP, Gelfand AE and Smith AFM (1992) Hierarchical Bayesian analysis of change points problems. Journal of the Royal Statistical Society. Series C (Applied Statistics), 41, 389-405.

Casella G, Girón FJ, Martínez ML and Moreno E (2009) Consistency of Bayesian procedures for variable selection. The Annals of Statistics, 37, 1207-28.

Coenen S, Adriaenssens N, Versporten A, Muller A, Minalu G, Faes C, Vankerckhoven V, Aerts M, Hens N, Molenberghs G and Goossens H; ESAC Project Group (2011) European Surveillance of Antimicrobial Consumption (ESAC): outpatient use of tetracyclines, sulphonamides and trimethoprim, and other antibacterials use in Europe (1997-2009). Journal of Antimicrobial Chemotherapy, 66 Suppl. 6, vi57-vi70.

Davey P, Ferech M, Ansari F, Muller A and Goossens H (2008) Outpatient antibiotic use in the four administrations of the UK: cross-sectional and longitudinal analysis. Journal of Antimicrobial Chemotherapy, 62, 1441-47.

Dominicus A, Ripatti S, Pedersen NL and Palmgren J (2008) A random change-point model for assessing variability in repeated measures of cognitive function. Statistics in Medicine, 27, 5786-98.

Gelman A, Carlin JB, Stern HS and Rubin DB (2004) Bayesian data analysis (2nd edn). Florida: Chapman \& Hall/CRC.

Ghosh P and Vaida F (2007) Random changepoint modelling of HIV immunologic responses. Statistics in Medicine, 26, 2074-87.

Goossens H, Ferech M, Vander Stichele RH and Elseviers M (2005) Outpatient antibiotic use in Europe and association with resistance: a crossnational database study. Lancet, 365, 579-87.

Hall CB, Lipton RB, Sliwinski M and Stewart WF (2000) A change-point model for estimating the onset of cognitive decline in preclinical Alzheimer's disease. Statistics in Medicine, 19, 1511-66.

Hall CB, Ying J, Kuo L and Lipton RB (2003) Bayesian and profile likelihood change-point methods for modeling cognitive function over time. Computational Statistics \& Data Analysis, 42, 91-109.

Hens N, Kvitkovicova A, Aerts M, Hlubinka D, and Beutels P (2010) Modelling distortions in seroprevalence data using change-point fractional polynomials. Statistical Modelling, 10(2), 159-75.

Huttner B, Goossens H, Verheij T and Harbarth S (2010) Characteristics and outcomes of public campaigns aimed at improving the use of antibiotics in outpatients in high-income countries. The Lancet Infectious Diseases, 10(1), 17-31. 


\section{Girma Minalu et al.}

Jacqmin-Gadda H, Commenges D and Dartigues J (2006) Random changepoint model for joint modeling of cognitive decline and dementia. Biometrics, 62, 254-60.

Kiuchi AS, Hartigan JA, Holford TR, Rubinstein P and Stevens CE (1995) Change-points in the series of T4 counts prior to AIDS. Biometrics, 51, 236-48.

Lange N, Carlin BP and Gelfand AE (1992) Hierarchical bayes models for the progression of HIV infection using longitudinal CD4 T-cell numbers (with discussion). Journal of American Statistical Association, 87, 615-32.

Lindley DV (1957) A statistical paradox. Biometrika, 44, 187-92.

Minalu G, Aerts M, Coenen S, Versporten A et al. (2011) Application of mixed-effects models to study the country-specific outpatient antibiotic use in Europe: a tutorial on longitudinal data analysis. Journal of Antimicrobial Chemotherapy, 66 Suppl. 6, vi79-vi87.

Muggeo VMR (2003) Estimating regression models with unknown break-points. Statistics in Medicine, 22, 3055-71.

Mulder J, Klugkist I, van de Schoot R, Meeus WHJ, Selfhout M and Hoijtink H (2009) Bayesian model selection of informative hypotheses for repeated measurements. Journal of Mathematical Psychology, 53, 530-46.
Ntzoufras I (2009) Bayesian modeling using WinBUGS. New York: John Wiley \& Sons.

Overstall AM and Forster JJ (2010) Default Bayesian model determination methods for generalized mixed models.

Computational Statistics and Data Analysis, 54, 3269-88.

Pastor R and Guallar E (1998) Use of two-segmented logistic regression to estimate change-points in epidemiologic studies. American Journal of Epidemiology, 148 (7), 631-42.

Plummer M (2008) Penalized loss functions for Bayesian model comparison. Biostatistics, 9(3), 523-39.

Slate EH and Turnbull BW (2000) Statistical models for longitudinal biomarkers of disease onset. Statistics in Medicine, 19, 617-37.

Smith AFM (1975) A Bayesian approach to inference about a change-point in a sequence of random variables. Biometrika. 62, 407-16.

Spiegelhalter DJ, Best NG, Carlin BP and Linde A (2002) Bayesian measures of model complexity and fit. Journal of the Royal Statistical Society, Series B, 64(4), 583-639.

Sturtz S, Ligges U and Gelman A (2005) R2WinBUGS: A Package for Running WinBUGS from R. Journal of Statistical Software, 12(3), 1-16. 